\title{
Online Algorithm for Time Series Prediction Based on Support Vector Machine Philosophy
}

\author{
J.M. Górriz ${ }^{1}$, C.G. Puntonet ${ }^{2}$, and M. Salmerón ${ }^{2}$ \\ 1 E.P.S. Algeciras, Universidad de Cádiz, \\ Avda. Ramón Puyol s/n, 11202 Algeciras Cádiz, Spain \\ juanmanuel.gorriz@uca.de \\ 2 E.S.I., Informática, Universidad de Granada \\ C/ Periodista Daniel Saucedo, 69042 Granada, Spain \\ \{carlos, moises\}@atc.ugr.es
}

\begin{abstract}
In this paper we prove the analytic connection between Support Vector Machines (SVM) and Regularization Theory (RT) and show, based on this prove, a new on-line parametric model for time series forecasting based on Vapnik-Chervonenkis (VC) theory. Using the latter strong connection, we propose a regularization operator in order to obtain a suitable expansion of radial basis functions (RBFs) and expressions for updating neural parameters. This operator seeks for the "flattest" function in a feature space, minimizing the risk functional. Finally we mention some modifications and extensions that can be applied to control neural resources and select relevant input space.
\end{abstract}

\section{Introduction}

The purpose of this work is twofold. It introduces the foundations of SVM 4 and its connection with RT [1. Based on this connection we show the new on-line algorithm for time series forecasting. SVMs are learning algorithms based on the structural risk minimization principle 2] (SRM) characterized by the use of the expansion of support vector (SV) "admissible" kernels and the sparsity of the solution. They have been proposed as a technique in time series forecasting [3] and they have faced the overfitting problem, presented in classical neural networks, thanks to their high capacity for generalization. The solution for SVM prediction is achieved solving the constrained quadratic programming problem. thus $\mathrm{SV}$ machines are nonparametric techniques, i.e. the number of basis functions are unknown before hand. The solution of this complex problem in real-time applications can be extremely uncomfortable because of high computational time demand.

SVMs are essentially Regularization Networks (RN) with the kernels being Green's function of the corresponding regularization operators 4. Using this connection, with a clever choice of regularization operator (based on SVM philosophy), we should obtain a parametric model being very resistant to the overfitting problem. Our parametric model is a Resource allocating Network [5] 
characterized by the control of neural resources and by the use of matrix decompositions, i.e. Singular Value Decomposition (SVD) and QR Decomposition with pivoting to input selection and neural pruning [6].

We organize the essay as follows. SV algorithm and its analytic connection to RT Theory will be presented in section 2. The new on-line algorithm will be compare to a previous version of it and to the standard SVM in section 4 Finally we state some conclusions in section 5

\section{Analytic Connection between SVM and RT}

The SV algorithm is a nonlinear generalization of the generalized portrait developed in the sixties by Vapnik and Lerner in [10]. The basic idea in SVM for regression and function estimation, is to use a mapping $\Phi$ from the input space $\mathcal{X}$ into a high dimensional feature space $\mathcal{F}$ and then to apply a linear regression. Thus the standard linear regression transforms into:

$$
f(x)=\langle\omega \cdot \Phi(x)\rangle+b .
$$

where $\Phi: \mathcal{X} \rightarrow \mathcal{F}, b$ is a bias or threshold and $\omega \in \mathcal{F}$ is a vector defining the function class. The target is to determinate $\omega$, i.e. the set of parameters in the neural network, minimizing the regularizated risk expressed as:

$$
R_{\text {reg }}[f]=R_{e m p}[f]+\lambda\|\omega\|^{2} .
$$

thus we are enforcing "flatness" in feature space, that is we seek small $\omega$. Note that equation 2 is very common in RN with a certain second term. SVM algorithm is a way of solving the minimization of equation 2 which can be expressed as a quadratic programming problem using the formulation stated in 11]:

$$
\text { minimize } \frac{1}{2}\|\omega\|^{2}+C \sum_{i=1}^{\ell}\left(\xi_{i}+\xi_{i}^{*}\right) .
$$

given a suitable Loss function $L(\cdot) \sqrt{1}$, a constant $C \geq 0$ and with slack variables $\xi_{i}, \xi_{i}^{*} \geq 0$. The optimization problem is solve constructing a Lagrange function by introducing dual variables, using equation 3 and the selected loss function. Once it is uniquely solved, we can write the vector $\omega$ in terms of the data points as follows:

$$
\omega=\sum_{i=1}^{\ell}\left(\alpha_{i}-\alpha_{i}^{*}\right) \Phi\left(x_{i}\right) .
$$

where $\alpha_{i}, \alpha_{i}^{*}$ are the solutions of the mentioned quadratic problem. Once this problem, characterized by a high computational demand 2, is solved we use equation 4 and [1] obtaining the solution in terms of dot products:

$$
f(x)=\sum_{i=1}^{\ell}\left(\alpha_{i}-\alpha_{i}^{*}\right)\left\langle\Phi\left(x_{i}\right) \cdot \Phi(x)\right\rangle+b .
$$

\footnotetext{
${ }^{1}$ For example Vapnik's $\epsilon$ insensitive loss function [11.

2 This calculation must be compute several times during the process
} 
At this point we use a trick to avoid computing the dot product in high dimensional feature space in equation 5, replacing it by a kernel function that satisfies Mercer's condition. Mercer's Theorem [12 guarantees the existence of this kernel function:

$$
f(x)=\sum_{i=1}^{\ell} h_{i} \cdot k\left(x_{i}, x\right)+b .
$$

where $h_{i} \equiv\left(\alpha_{i}-\alpha_{i}^{*}\right)$ and $k\left(x_{i}, x\right)=\left\langle\Phi\left(x_{i}\right) \cdot \Phi(x)\right\rangle$. Finally we note, regarding the sparsity of the SV expansion 5, that only the elements satisfying $\left|f\left(x_{i}\right)-y_{i}\right| \geq \epsilon$, where $\epsilon$ is the standard deviation of $f\left(x_{i}\right)$ from $y_{i}$ (see selected loss function), have nonzero Lagrange multipliers $\alpha_{i}, \alpha_{i}^{*}$. This can be proved applying KarushKuhn-Tucher (KKT) conditions [13] to the SV dual optimization problem.

\subsection{Regularization Theory}

RT appeared in the methods for solving ill posed problems [1]. In RN we minimize a expression similar to equation 2 However, the search criterium is enforcing smoothness (instead of flatness) for the function in input space (instead of feature space). Thus we get:

$$
R_{r e g}[f]=R_{e m p}[f]+\frac{\lambda}{2}\|\hat{P} f\|^{2} .
$$

where $\hat{P}$ denotes a regularization operator in the sense of [1], mapping from the Hilbert Space $H$ of functions to a dot product Space $D$ such as $\langle f, g\rangle \quad \forall f, g \in H$ is well defined. Applying Fréchet's differentia 3 to equation 7 and the concept of Green's function of $\hat{P}^{*} \hat{P}$ :

$$
\hat{P}^{*} \hat{P} \cdot G\left(x_{i}, x_{j}\right)=\delta\left(x_{i}-x_{j}\right) .
$$

(here $\delta$ denotes the Dirac's $\delta$, that is $\left\langle f, \delta\left(x_{i}\right)\right\rangle=f\left(x_{i}\right)$ ), we get [6]:

$$
f(x)=\lambda \sum_{i=1}^{\ell}\left[y_{i}-f\left(x_{i}\right)\right]_{\epsilon} \cdot G\left(x, x_{i}\right) .
$$

The correspondence between SVM and RN (equations 6] and 9) is proved if and only if the Green's function $G$ is an "admissible" kernel in the terms of Mercer's theorem [12, i.e. we can write $G$ as:

$$
G\left(x_{i}, x_{j}\right)=\left\langle\Phi\left(x_{i}\right), \Phi\left(x_{j}\right)\right\rangle \quad \text { with } \quad \Phi: x_{i} \rightarrow(\hat{P} G)\left(x_{i}, .\right) .
$$

Prove: Minimizing $\|\mathbf{P} f\|^{2}$ can be expressed as:

$$
\|\mathbf{P} f\|^{2}=\int d x(\mathbf{P} f)^{2}=\int d x f(x) \mathbf{P}^{*} \mathbf{P} f(x)
$$

we can expand $\mathrm{f}$ in terms of Green's function associated to $\mathbf{P}$, thus we get:

\footnotetext{
$\overline{{ }^{3} \text { Generalized }}$ differentiation of a function: $d R[f]=\left[\frac{d}{d \rho} R[f+\rho h]\right]$, where $h \in H$.
} 


$$
\begin{aligned}
& \|\mathbf{P} f\|^{2}=\sum_{i, j}^{N} h_{i} h_{j} \int d x G\left(x, x_{i}\right) \mathbf{P}^{*} \mathbf{P} G\left(x, x_{j}\right) \\
& =\sum_{i, j}^{N} h_{i} h_{j} \int d x G\left(x, x_{i}\right) \delta\left(x-x_{j}\right) \\
& =\sum_{i, j}^{N} h_{i} h_{j} G\left(x_{j}, x_{i}\right)
\end{aligned}
$$

then only if $G$ is Mercer Kernel it correspond to a dot product in some feature space. Then minimizing 7 is equivalent to minimize 2 .

A similar prove of this connection can be found in [4]. Hence given a regularization operator, we can find an admissible kernel such that SV machine using it will enforce flatness in feature space and minimize the equation 7 Moreover, given a SV kernel we can find a regularization operator such that the SVM can be seen as a RN.

\section{Online Endogenous Learning Machine Using Regularization Operators}

In this section we show a new on-line RN based on "Resource Allocating Network" algorithms (RAN) 45] which consist of a network using RBFs, a strategy for allocating new units (RBFs), using two part novelty condition [5]; input space selection and neural pruning using matrix decompositions such as SVD and QR with pivoting [6]; and a learning rule based on SRM as discuss in the previous sections. The pseudo-code of the new on-line algorithm is presented in section 3.1. Our network has 1 layer as is stated in equation 6. In terms of RBFs the latter equation can be expressed as:

$$
f(x)=\sum_{i=1}^{N(t)} h_{i} \cdot \exp \left(-\frac{\left\|x(t)-x_{i}(t)\right\|^{2}}{2 \sigma_{i}^{2}(t)}\right)+b .
$$

where $N(t)$ is the number of neurons, $x_{i}(t)$ is the center of neurons and $\sigma_{i}(t)$ the radius of neurons, at time "t".

In order to minimize equation 7 we propose a regularization operator based on SVM philosophy. We enforce flatness in feature space, as described in section 2 using the regularization operator $\|\hat{P} f\|^{2} \equiv\|\omega\|^{2}$, thus we get:

$$
R_{r e g}[f]=R_{e m p}[f]+\frac{\lambda}{2} \sum_{i, j=1}^{N(t)} h_{i} h_{j} k\left(x_{i}, x_{j}\right) .
$$

We assume that $R_{e m p}=(y-f(x))^{2}$ we minimize equation 14 adjusting the centers and radius (gradient descend method $\Delta \chi=-\eta \frac{\partial R[f]}{\partial \chi}$, with simulated annealing [14):

$$
\Delta x_{i}=-2 \frac{\eta}{\sigma_{i}}\left(x-x_{i}\right) h_{i}(f(x)-y) k\left(x, x_{i}\right)+\alpha \sum_{i, j=1}^{N(t)} h_{i} h_{j} k\left(x_{i}, x_{j}\right)\left(x_{i}-x_{j}\right) .
$$

and

\footnotetext{
${ }^{4}$ The principal feature of these algorithms is sequential adaptation of neural resources.
} 


$$
\Delta h_{i}=\tilde{\alpha}(t) f\left(x_{i}\right)-\eta(f(x)-y) k\left(x, x_{i}\right) .
$$

where $\alpha(t), \tilde{\alpha}(t)$ are scalar-valued "adaptation gain", related to a similar gain used in the stochastic approximation processes [15], as in these methods, it should decrease in time. The second summand in equation 15 can be evaluated in several regions inspired by the so called "divide-and-conquer" principle and used in unsupervised learning, i.e. competitive learning in self organizing maps [16] or in SVMs experts [17. This is necessary because of volatile nature of time series, i.e. stock returns, switch their dynamics among different regions, leading to gradual changes in the dependency between the input and output variables [18]. Thus the super-index in the latter equation is redefined as:

$$
N_{c}(t)=\left\{s_{i}(t):\left\|x(t)-x_{i}(t)\right\| \leq \rho\right\} .
$$

that is the set of neurons close to the current input.

\subsection{Program Pseudo-Code}

The structure of the algorithm is shown below as pseudo-code:

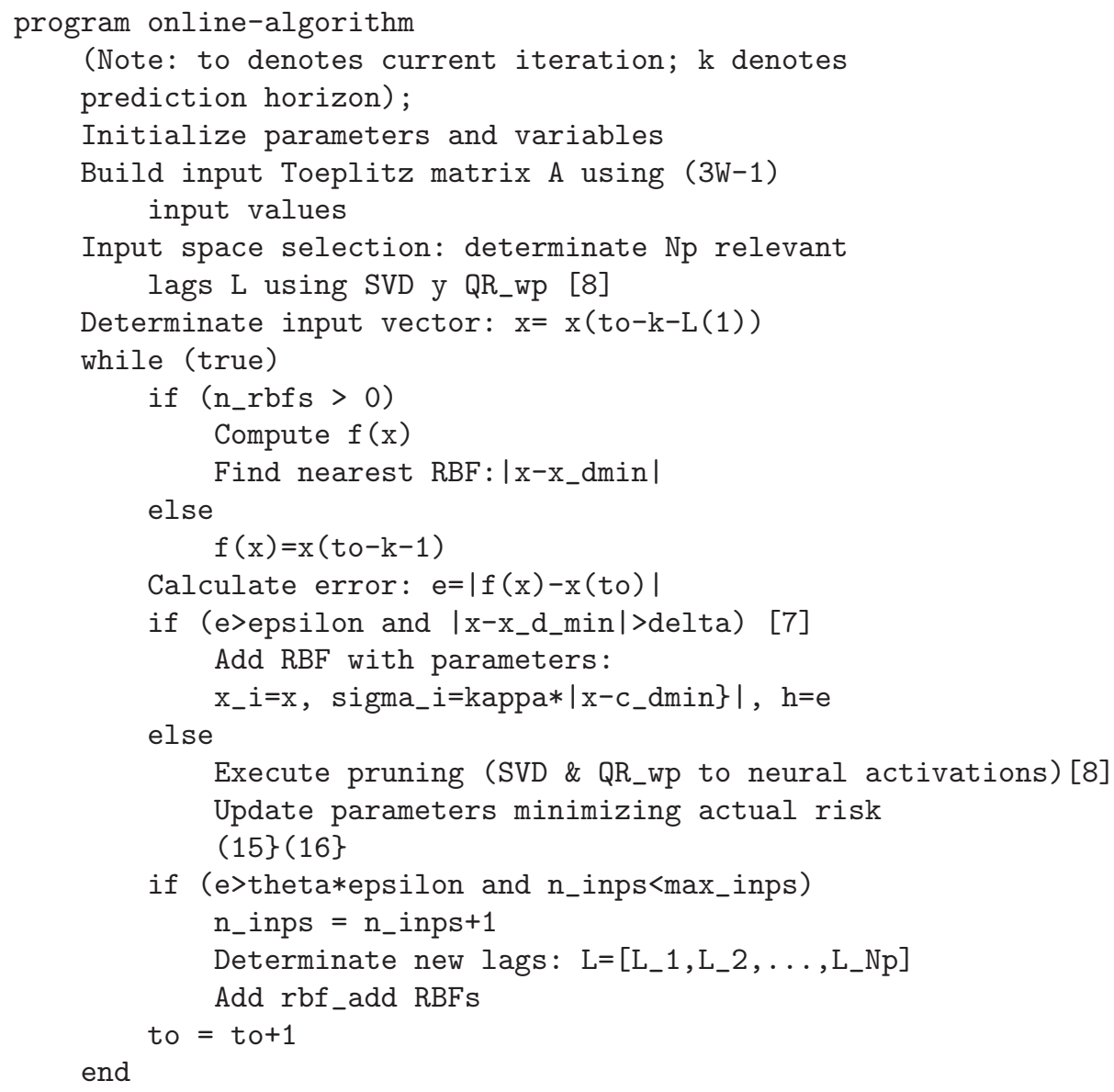




\section{Experiments}

The application of our network is to predict complex time series. We choose the high-dimensional chaotic system generated by the Mackey-Glass delay differential equation:

$$
\frac{d x(t)}{d t}=-b \cdot x(t)+a \cdot \frac{x(t-\tau)}{1+x^{10}(t-\tau)} .
$$

with $b=0.1, a=0.2$ and delay $t_{d}=17$. This equation was originally presented as a model of blood regulation [19] and became popular in modelling time series benchmark. We add two modifications to equation 18 Zero-mean gaussian noise with standard deviation equal to $1 / 4$ of the standard deviation of the original series and dynamics changes randomly in terms of delay (between 100-300 time steps) $t_{d}=17,23,30$.

We integrated the chaotic model using MatLab software on a Pentium III at $850 \mathrm{MHZ}$ obtaining 2000 patterns. For our comparison we use 100 prediction results from SVM_online (presented in this paper), standard SVM (with $\epsilon$-insensitive loss) and NAPA_PRED (RAN algorithm using matrix decompositions being one of the best on-line algorithms to date [6]).

Table 1. Evolution of NRMSE (Normalized Root Mean Square Error). Xth-S detones the Xth-step prediction NRMSE on the test set.

\begin{tabular}{llllll}
\hline Method & 1st-S & 25 th-S & 50 th-S & 75 -th & 100 th-S \\
\hline NAPA_PRED & 1.1982 & 0.98346 & 0.97866 & 0.91567 & 0.90985 \\
Standard SVM & 0.7005 & 0.7134 & 0.7106 & 0.7212 & 0.7216 \\
SVM_online & 0.7182 & 0.71525 & 0.71522 & 0.72094 & 0.7127 \\
\hline
\end{tabular}

Clearly there's a remarkable difference between previous on-line algorithm and SVM philosophy. Standard SVM and SVM_online achieve similar results for this set of data at the beginning of the process. In addition, there's is noticeable improvement in the last iterations because of the volatile nature of the series. The change in time delay $t_{d}$, leads to gradual changes in the dependency between the input and output variables and, in general, it's hard for a single model including SVMs to capture such a dynamic input-output relationship inherent in the data. Focussing our attention on the on-line algorithm, we observe the better performance of the new algorithm as is shown in table 2

\section{Conclusions}

Based on SRM and the principle of "divide and conquer" , a new online algorithm is developed by combining SVM and SOM using a resource allocating network and matrix decompositions. Minimizing the regularizated risk functional, using an operator the enforce flatness in feature space, we build a hybrid model 
that achieves high prediction performance, comparing with the previous on-line algorithms for time series forecasting. This performance is similar to the one achieve by SVM but with lower computational time demand, essential feature in real-time systems. The benefits of SVM for regression choice consist in solving a - uniquely solvable - quadratic optimization problem, unlike the general RBF networks, which requires suitable non-linear optimization with danger of getting stuck in local minima. Nevertheless the RBF networks used in this paper, join various techniques obtaining high performance, even under extremely volatile conditions, since the level of noise and the change of delay operation mode applied to the chaotic dynamics was rather high.

Table 2. Figures: 1) The predicted and actual values in Noisy Mackey-Glass data for online algorithms. 2) Evolution of the input space dimension in SVM_online vs. NAPA_PRED. The higher capacity of generalization controls input space dimension. 3) Evolution of the neural resources in SVM_online vs. NAPA_PRED and number of neighboring neurons. 4) Noisy Mackey-Glass Series with changing operation mode.
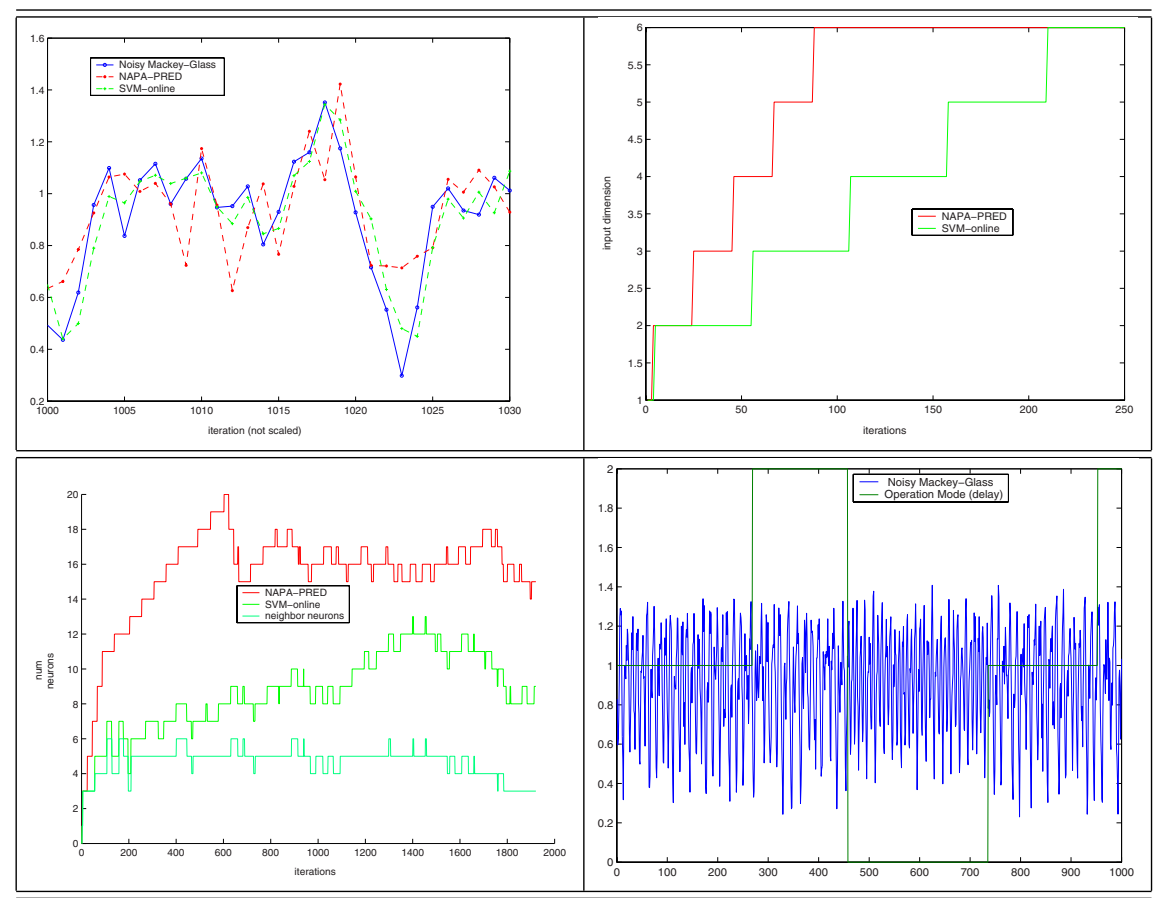

\section{References}

1. Tikhonov, A.N., Arsenin, V.Y.:, Solutions of Ill-Posed Problems, Winston. Washington D.C., U.S.A. Berlin Heidelberg New York (1997) 415-438 
2. Vapnik, V., Chervonenkis, A.: Theory of Pattern Recognition [in Russian]. Nauka, Moscow (1974).

3. Muller, K.R, Smola A.J., Ratsch, G., Scholkopf, B., Kohlmorgen, J: Using Support Vector Machines for time series prediction, In Advances in kernel Methods- Spport Vector Learning, MIT Press, Cambridge, MA. (1999) 243-254

4. Smola, A.J., Scholkopf, B., Muller, K.R: The connection between regularization operators and support vector kernels. Neural Networks, 11, 637-649

5. Platt, J.: A resource-allocating network for function interpolation. Neural Computation, 3, (1991) 213-225

6. Salmerón-Campos, M.: Predicción de Series Temporales con Rede Neuronales de Funciones Radiales y Técnicas de Descomposición Matricial. PhD Thesis, University of Granada, DEpartamento de Arquitectura y Tecnología de Computadores (2001)

7. Kolmogorov, A.N.: On the representation of continuous fucntions of several variables by superposition of continuos fucntions of one variable and addition. Dokl. Akad. Nauk USSR, vol 114, 953-956 (1957)

8. Muller, K.R, Mika, S., Ratsch, G., Tsuda, K., Scholkopf, B.: An Introduction to Kernel-Based Learning Algorithms. IEEE Transactions on neural Networks, vol 12, num 2, 181-201 (2001)

9. Poggio, T., Girosi, F.,: Regularization algorithms for learning that are equivalent to multilayer networks. Science, vol 247, 978-982 (1990)

10. Vapnik, V., Lerner, A.: Pattern Recognition using Generalized Portrait Method. Automation and Remote Control, vol 24, issue 6,(1963)

11. Vapnik, V.: Statistical Learning Theory. Wiley, N.Y. (1998)

12. Mercer, J.: Functions of positive and negative type and their connection with the theory of integral equations. Philos. Trans. Roy. Soc. London, num 209, vol A, 415-446 (1909)

13. Kuhn, H.W.,Tucker, A.W.: Nonlinear Programming. In $2^{\text {nd }}$ Symposium on Mathematical Statistics and Probabilistics, University of California Press, 481-492 (1951)

14. Kirkpatrick, S., Gelatt, C.D., Vecchi, M.P.: Optimization by Simulated Annealing. Science, vol 220, num 4598, 671-680, (1983)

15. Kushner, H.J., Yin, G.:Stochastic Approximation Algorithms and Applications. Springer-Verlag, New York, U.S.A. (1997)

16. Kohonen, T.: The Self-Organizing Map. Proceedings of the IEEE, num 9, vol 78, 1464-1480 (1990)

17. Cao, L.: Support Vector Machines Experts for Time Series Forecasting- Neurocomputing, vol 51, 321-339 (2003)

18. Górriz, J.M. : Algorítmos Híbridos para la Modelización de Series Temporales con Técnicas AR-ICA. In Press PhD Thesis, University of Cádiz (2003)

19. Mackey, M.C., Glass, L., Science, 197 287-289 (1977) 